\title{
Inter-Pixel Euclidean Paths for Image Analysis
}

\author{
Jean-Pierre BRAQUELAIRE and Luc BRUN and Anne VIALARD
}

\author{
LaBRI ${ }^{2}$, Université Bordeaux I \\ 351, cours de la Libération \\ 33405, Talence Cedex - France \\ \{Jean-Pierre.Braquelaire|Luc.Brun|Anne.Vialard\}@labri.u-bordeaux.fr
}

\begin{abstract}
Inter-pixel boundaries provide a robust and consistent description of segmented images but have a poor visual aspect, especially when being enlarged. Approximation curve are sometimes used to smooth discrete boundaries but they do not provide error free reconstruction and may be uneasy to use in this context. In this paper we show the advantages of using Euclidean paths in order to smooth inter-pixel boundaries and we demonstrate the interest of inter-pixel Euclidean paths for the purpose of image segmentation and analysis.
\end{abstract}

\section{Introduction}

The most common way to implicitly represent the regions of a 2D discrete image is the boundary representation. Two main approaches have been used. Roughly speaking, the first one [Fre61] consists in drawing the boundary "on" the pixels while the second one[BF70] consists in drawing it "between" the pixels. According to the terminology of inter-pixel boundary used by Fiorio[Fio95] we call this second approach the inter-pixel oriented approach, and thus the first one the pixel oriented approach.

Region boundaries present many interests from the point of view of both image synthesis and image analysis (extraction of geometrical features, image understanding, image compositing, etc.). Discrete boundaries provide an exact reconstruction of the domain of the image. On the other hand, by reason of their discrete nature, they have many geometrical drawbacks: aliased aspect, not differentiable, ill-adapted to geometrical transformations, etc. Several approaches have been developed in order to infer Euclidean boundaries from the discrete ones. Unfortunately these Euclidean boundaries have not a canonical form and do not provide an error free reconstruction of the region domain. In order to overcome this problem Braquelaire and Vialard have introduced Euclidean paths [BV95] which are a compromise between Euclidean and discrete boundaries.

The aim of this paper is to demonstrate the interest of mixing together both approaches of inter-pixel representation and Euclidean paths for the purpose of image segmentation and analysis. We consider images partitioned into

${ }^{1}$ Laboratoire Bordelais de Recherche en Informatique, Unité de Recherche 1304 Associée au Centre National de la Recherche Scientifique. This work is granted by the Région Aquitaine and the C.N.R.S. 
4-connected regions. The boundary of a region is made of one or more interpixel 4-connected paths. The boundaries of the whole segmented image define a set of underlying topological maps [Dom92] providing a consistent topological framework. This model has been used as the kernel of a segmentation platform currently under development [BB96]. Euclidean paths have been implemented in this software, providing an interactive and automatic smoothing of boundaries. Such a smoothed boundary can be enlarged without staircase enhancement. Moreover since the discretization of the smoothed boundary produces exactly the discrete boundary, the smoothed boundary can be used to interactively extract geometric features and to drive segmentation steps.

In section 2 we briefly recall some advantages of the inter-pixel oriented approach and we describe the boundary model we have used. In section 3 we compare the Euclidean paths approach with the curve approximation ones. In section 4 we describe how to adapt Euclidean paths to a boundary graph. Finally several examples are given and discussed in the last section.

\section{Pixel boundaries versus inter-pixel boundaries}

The older approach used to represent the boundary of a discrete region is the pixel oriented approach where the boundary elements are defined in the domain of the image [Fre61, Mer73, Fre74, Pav82, RK82, CL90]. According to conventions the boundary points are either points of the region or of its complementary. The inside pixel boundary of a 4-connected region $r$ is the subset of its points having at least one 8-neighbor not belonging to $r$, and its outside pixel boundary is the set of pixels not belonging to $r$ but having a 4-neighbor in $r$. Finally, the boundary of a region induces one or more closed discrete curves (one plus the number of holes) also called contours.

A well known disadvantage of pixel oriented boundaries is that contours are not necessarily simple. Conversely, a simple curve drawn in the image space do not satisfy Jordan's theorem. Moreover two adjacent objects do not share the same boundary element. This drawback can be seen as a minor one when considering the restricted case of a foreground object laying on a background since it is generally useless to deal with the contour of the background. On the other hand it is a major drawback when considering an image decomposed into several adjacent objects.

An alternative to pixel boundaries is inter-pixel boundaries which has been introduced in 1970 by Brice and Fennema [BF70] as a data structure for implementing grouping segmentation. The pixels are considered as squares, two adjacent pixels sharing a vertical or an horizontal edge. A boundary is a sequence of pixel edges encoded by a sequence of points located at pixel corners. From the earlier eighties several theoretical approaches have been developed introducing discrete topology. The solution generally adopted is to extend the concept of pixel [KR89, KKM90b, KKM90a, Kov89, Bie90, Fra91, Fra95, AAF95] to allow a consistent definition of open and closed sets in $\mathbb{Z}^{2}$. These approaches provided a rigorous topological framework for digital boundaries. Another ap- 
proach has been developed by Braquelaire and Domenger consisting in associating the inter-pixel boundaries of a segmented image with a set of topological maps [BD91, Dom92]. This approach is similar to the one proposed by Morse [Mor69] and Gangnet [GVT91].

Let us give the following definitions [BD96a]. The half-integer plane, denoted by $P_{\frac{1}{2}}$, is the plane resulting from the translation of the usual discrete plane $\mathbb{Z}^{2}$ by $\left(\frac{1}{2}, \frac{1}{2}\right)$ :

$$
P_{\frac{1}{2}}=\left\{\left(i+\frac{1}{2}, j+\frac{1}{2}\right), \text { with }(i, j) \in \mathbb{Z}^{2}\right\}
$$

The half-integer plane is also called the boundary plane. If $p=\left(x_{p}, y_{p}\right)$ is a point of the image plane $\mathbb{Z}^{2}$ and $p^{\prime}=\left(x_{p}^{\prime}, y_{p}^{\prime}\right)$ is a point of the boundary plane then $p$ and $p^{\prime}$ are half-neighbors if $\left|x_{p}-x_{p}^{\prime}\right|=\left|y_{p}-y_{p}^{\prime}\right|=\frac{1}{2}$. Each point of the image plane has four half-neighbors in the boundary plane and conversely. Two adjacent points of the boundary plane share exactly two halfneighbors in the image plane. Each point of the boundary plane having two or more half-neighboring points belonging to different regions of a segmented image is a boundary point. Two adjacent boundary points are linked if their common half-neighbors belong to different regions. A contour is a sequence of boundary points $b_{1}, b_{2}, \ldots, b_{n}$ with $n>1$ and such that:

1. $b_{i}$ is linked to $b_{i}+1, \forall i$ with $1 \leq i<n$.

2. $b_{i} \neq b_{j}, \forall i, j$ with $i \neq j$.

A closed contour is a sequence of boundary points $b_{1}, b_{2}, \ldots, b_{n}, b_{1}$, such that

1. $b_{1}, b_{2}, \ldots, b_{n}$ is a contour.

2. $b_{n}$ is linked to $b_{1}$.

Any boundary point linked to more than two other ones is a natural node. A segment is a maximal contour without node. An augmented segment is a segment augmented with both its linked nodes.

By embedding both the boundary plane and the image plane into the Khalimsky's plane [KKM90a] it can be verified that a closed single path of $P_{\frac{1}{2}}$ satisfy Jordan's theorem and that the boundaries of a segmented image define a unique set of planar maps augmented with an inclusion relation [BD96a]. These maps are canonically induced by the decomposition of the boundary into nodes, corresponding to map vertices, and segments, corresponding to map edges.

Remark. In order to fit with the usual definition of topological maps [Cor75] an arbitrary node has to be added on each closed contour without natural node. But this aspect can be ignored for the purpose of this paper. Thus, in order to simplify this presentation we shall consider in the following that a single loop without node is also a segment.

To sum up boundaries defined in $P_{\frac{1}{2}}$ have the following properties: 
1. The boundaries of the segmented image are decomposed into nodes and segments. A segment is either a single loop or a single path joining two nodes.

2. Each connected set of boundaries defines a planar map.

3. The boundary of a region is a set of simple closed 4-connected paths.

4. Two adjacent regions share one or more augmented segments which constitute their common boundary.

5. This decomposition is canonical.

\section{Smoothing discrete paths}

As sketched above a discrete contour has many geometrical drawbacks and it is interesting to build a Euclidean curve as close as possible to the discrete one. Several criteria can be considered to evaluate the quality of a Euclidean boundary. We have retained the following ones:

1. The visual aspect (smoothness, preservation of lines and angles, etc.). This feature is of course subjective.

2. The uniqueness (does the method produce a unique Euclidean boundary from a discrete one ?). This feature is important for an automatic construction of the Euclidean curve.

3. The reconstruction quality. A reconstruction is error free if it produces exactly the same set of pixels than the one defined by the discrete boundary. An error free reconstruction is a strong condition of closeness of both Euclidean and discrete curves.

4. The complexity. It includes the computational complexity of the construction and the complexity of use of the method.

A fifth aspect which is the extraction of geometrical features such as perimeter or tangent orientations, can also be considered to estimate the quality of a Euclidean boundary. This aspect is developed in another work [Via96].

The usual approach used to build a Euclidean boundary from a discrete one is an approximation approach. The problem consists in finding the curve from a given family of curves which minimizes some error criterion. The vectorization approach is based on the construction of a sequence of real straight lines of which a possible discretization is the discrete contour. Several techniques exists but none of these methods provides a canonical vectorization and the result may highly depend on the boundary point on which the vectorization is initiated. It is also possible to use higher degree approximation curves such as splines. Each boundary point can be used as a control point of the spline curve. Experiments show us that to avoid oscillations it is necessary to use very high degree curves which does not preserve angular points. It is possible to reduce the set of control points to a subset of the discrete points but it is very difficult to do it automatically. Moreover splines cannot ensure an error free reconstruction. 
Another model called Euclidean paths has been proposed [BV95] to smooth discrete contours. This approach is intermediate between a purely discrete approach and a continuous one. A Euclidean path associated with a discrete path $\left(p_{1}, \ldots, p_{n}\right)$ is a sequence of Euclidean points $\left(\pi_{1}, \ldots, \pi_{n}\right)$ which verifies the following property:

$$
\forall k \in\{1, \ldots, n\}\left|x_{k}-i_{k}\right|<\frac{1}{2} \text { and }\left|y_{k}-j_{k}\right|<\frac{1}{2}
$$

with $\pi_{k}=\left(x_{k}, y_{k}\right)$ and $p_{k}=\left(i_{k}, j_{k}\right)$.

By rounding each coordinate of a point of a Euclidean path to the closest integer we get the related coordinate of the associated discrete path. Thus the reconstruction is obviously error free. We have shown that by using the definition of discrete lines given by Reveilles [Rev91] and by adapting the vectorization algorithm of Debled and Reveilles [DR92] it is possible to compute in a linear time a local tangent $\Delta(p)$ at each boundary point $p$ such that there exists a canonical projection of $p$ on $\Delta(p)$ which is a Euclidean point associated with $p$. This method provides a technique of construction of a family of Euclidean paths called Tangent driven Euclidean paths (see [BV95]).

To sum up Euclidean paths present the following advantages:

1. They provide a good visual aspect by removing jaggies and preserving angles (see for instance the example displayed in Figure 6).

2. The construction is canonical.

3 . The reconstruction is error free.

4. The complexity is in $O(l . n)$ where $l$ is the average length of the maximal discrete lines around each discrete point and $n$ the number of discrete points on the processed contour.

Finally Euclidean paths provide good estimation of perimeter,local tangents and curvature [Via96].

\section{Mixing both approaches}

In previous sections we have pointed out the advantages

- of the inter-pixel oriented approach compared with the pixel-oriented one for the representation of segmented image,

- of Euclidean paths compared with approximation curves for the boundary smoothing of a discrete region.

Both approaches can be combined in order to smooth the boundaries of a segmented image. We call inter-pixel Euclidean path (in short IPEP) the Euclidean path associated with an inter-pixel boundary.

Recall that the set of boundaries defines a partition of the segmented image into regions. The boundaries of these regions are made of nodes and segments defined in the $P_{\frac{1}{2}}$ plane. The set of nodes and segments which defines the boundary of a region $i$ is a closed curve $C_{i}$ defined in the $P_{\frac{1}{2}}$ plane. An IPEP $E C_{i}$ 
can be associated to each closed curve $C_{i}$. Thus, starting from a partition, of an image, we can build a set of closed curves $\left\{C_{1}, \ldots, C_{n}\right\}$, and a set of IPEP $\left\{E C_{1}, \ldots, E C_{n}\right\}$ based on these curves. The drawback of this method is that it does not preserve the consistency of the segmented image. As a matter of fact, let us suppose that two regions $i$ and $j$ are adjacent by a segment. Then we have $C_{i} \cap C_{j}=S_{i j}$, where $S_{i j}$ is a segment delimited by two nodes. Since $S_{i j}$ is shared by two regions, it has been traversed twice for computing $E C_{i}$ and $E C_{j}$. Thus two Euclidean points are associated with each point of $S_{i j}$. If all Euclidean points associated with the segment $S_{i j}$ are distinct we may have $E C_{i} \cap E C_{j}=\emptyset$ while $C_{i} \cap C_{j}=S_{i j} \neq \emptyset$. To overcome this inconsistency we associate with each boundary point $p$ the average of the Euclidean points associated to $p$. Since all Euclidean points associated to a boundary point verify equation 1, the mean of these points will verify the same equation and the set of points obtained by the averaging is still a set of Euclidean paths.

Thus, there is a one to one mapping between the boundaries and smoothed boundaries. Let us call boundary cells the open unit squares centered at boundary points. Since boundaries are 4-connected paths three adjacent boundary points may either be in line or make a right angle (see Figure 1). Each point of the IPEP boundary is located in the boundary cell centered at its associated boundary point. It follows that a boundary and its associated Euclidean path traverse exactly the same sequence of boundary cells. Thus if two boundary elements do not intersect then their associated IPEP do not intersect either. Conversely the construction described above ensures that intersection between boundary elements are preserved by smoothing. Hence the smoothing of the boundaries with Euclidean paths preserve the topology.
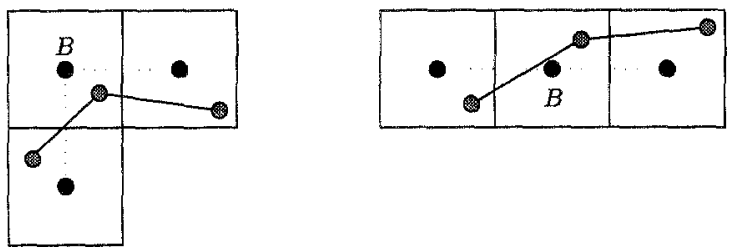

Fig. 1. The two possible neighboring configurations for a boundary point $B$ which is not a node.

Since Euclidean paths may also be defined for non-closed discrete curves a less time consuming method would be to compute the Euclidean path of each augmented segment. In this case, each segment will be traversed a single time and only one Euclidean path will be associated with each segment. The averaging would then be limited to nodes. This strategy has some drawbacks, which lead us to reject it. First, the object of interest of a segmented image are the regions which partition it. Thus a computation of IPEP based on regions is more meaningful than a computation of IPEP based on segments. Moreover, the computation of IPEP based on segments tends to deteriorate the smoothing effect around nodes. 


\section{Experiments and discussion}

In this section we present some examples of inter-pixel Euclidean paths on "real cases". All these images have been segmented and displayed by using an environment of segmentation that we are currently developing (this environment is described in several other works [BB96, BD96b]).

Consider the example displayed in Figure 2. The small square drawn on the upper right part corresponds to a pixel. There are two uniform grey regions with both the inter-pixel boundary and the associated Euclidean path. Remark first that both inter-pixel boundaries and Euclidean paths are symmetrical models of contour representation in the sense that a contour is never attached to a specific object of the image. An inter-pixel boundary separates equitably both objects sharing it. In the same way Euclidean paths provide a local best adjustment of all discrete lines composing the inter-pixel boundary. The inter-pixel Euclidean path boundary keeps a smooth appearance while keeping close to the inter-pixel boundary.

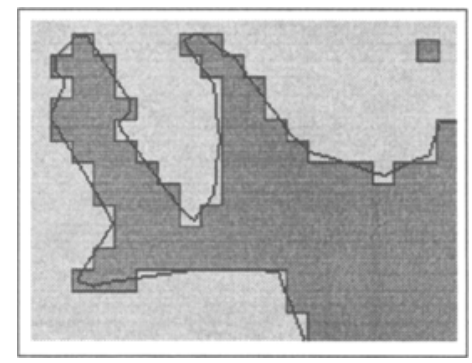

Fig. 2. The path drawn in black is the Euclidean path of the inter-pixel boundary separating both grey regions. The inter-pixel boundary is displayed in dark gray.

The left image displayed in Figure 3 shows a brain RMI on which is superimposed the inter-pixel boundaries resulting from a segmentation step. Of course inter-pixel boundaries cannot be displayed at scale 1 and the half-integer boundaries have been translated by $\left(\frac{1}{2}, \frac{1}{2}\right)$ to be drawn in the image plane. Nevertheless, even when drawn at scale 1, IPEP improve the visualization since they can be displayed in 8-connexity as real polylines. Pixels will then be drawn either on the inside or on the outside pixel boundary (see the right image of the same Figure).

Since the construction of Euclidean paths is canonical the smoothing of the boundaries does not need any user intervention. Moreover the algorithm being linear (see section 3 ) the smoothed boundary of a region can be drawn interactively. It allows us to switch between discrete and smoothed boundaries. When 


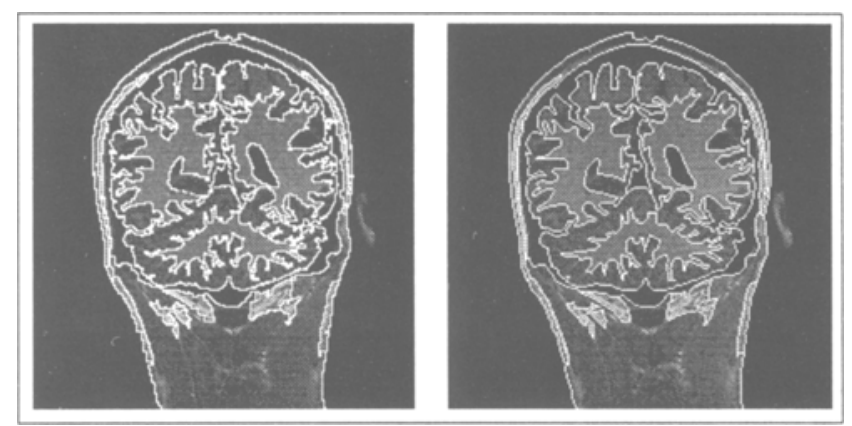

Fig. 3. This image results from a brain RMI. The size of the image is $230 \times 230$ pixels. On the left image are superimposed the discrete boundaries translated from the boundary plane and on the right one the discretization of the IPEP smoothed boundaries.

segmenting a small region it may be necessary to use an enlargement of the related area of the working image. In that case pixel boundaries can be displayed by drawing horizontal and vertical steps between pixels. Unfortunately the stair case effect is enhanced making the shape of the working region difficult to perceive. This drawback is removed when switching from discrete boundaries to IPEP smoothed boundaries (see Figure 4).

It is also possible to edit the contour by interactively addressing IPEP points with the mouse and working on the associated boundary points. The editing may be facilitated by superimposing both contours on the same image.

IPEP contours are very efficient for enlarging of discrete contours. In Figure 5 is shown an enlargement of a region of size $26 \times 19$ pixels in the original image (it is the small dark area located in the middle of the right hemisphere). The image of the region is displayed at scale 10. The IPEP smoothed boundary shown in the right image of the picture still has a visual aspect very close to the shape of the original region. There is a small irregularity on the upper-right part of the smoothed boundary. By comparing the discrete boundary with the smoothed one it appears that this irregularity corresponds to the pixel located on the second row and on the eighth column. This pixel has been aggregated to the dark region. The irregularity can be removed simply by changing interactively the assignment of this pixel. This example shows how IPEP smoothed boundary can help the user during an interactive segmentation and editing.

The last example results from the segmentation of a small color image of size $139 \times 200$ pixels (see Figure 6). Our segmentation environment allows the user to perform any segment and region removing at any time during the segmentation process. The segmented image has been edited to keep only the contour of the meloidae. The upper right drawing is the inter-pixel boundary at scale 1 . The smoothed boundary is the associated IPEP boundary at scale 4 . In figure 7 is visualized the upper horn at scale 10 with the corresponding part of the image also at scale 10 . 


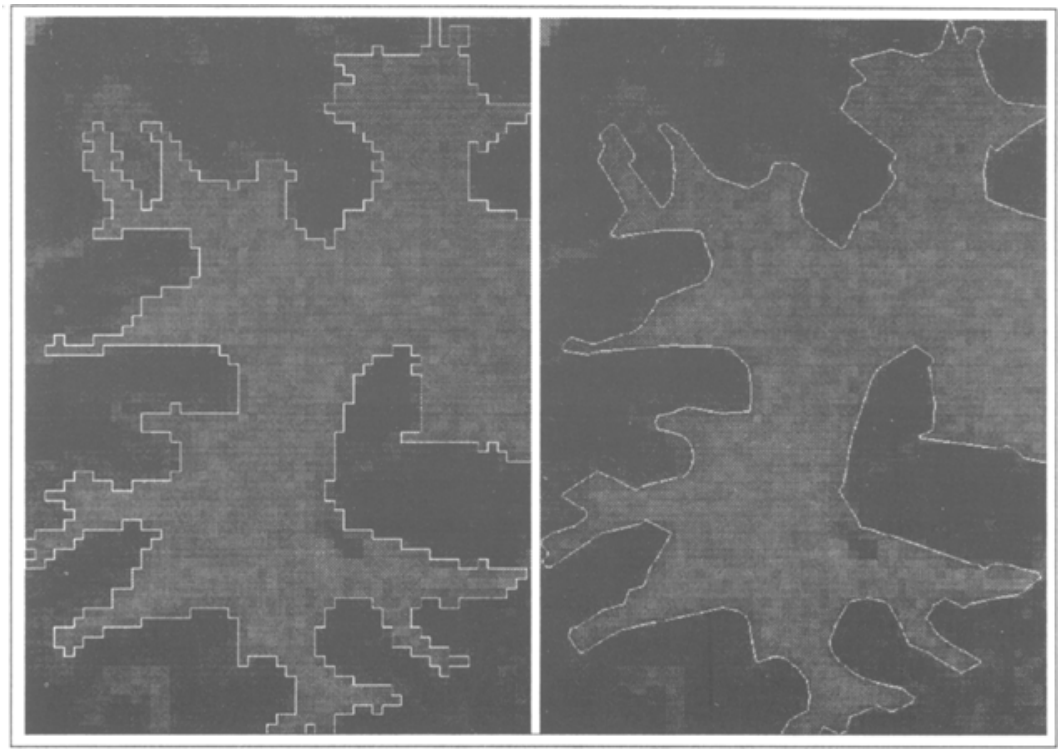

Fig. 4. The left picture shows an enlargement of the inter-pixel boundary of the upper part of the left hemisphere displayed in Figure 3. The right picture shows the associated IPEP smoothed boundary.

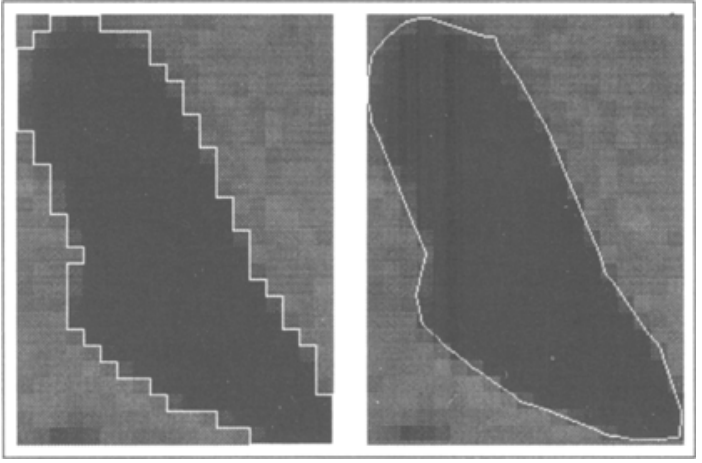

Fig. 5. This is an enlargement by 10 of the small dark area of the right hemisphere. The original size of the region is $26 \times 19$ pixels.

Finally IPEP smoothed boundaries provide an efficient framework for image analysis. The underlying topological maps allow a very efficient extraction of topological features and the Euclidean paths produces good results for geometrical features extraction ([Via96]). 


\section{Conclusion}

In this paper we have proposed to combine inter-pixel boundaries with Euclidean paths in order to improve image segmentation and we have described how to smooth a graph of boundaries. We have shown that IPEP smoothed boundaries present several important advantages: interactive and automatic construction, error free reconstruction, good visual aspect, efficiency for image enlargement. Moreover IPEP smoothed boundaries lay on the same unit cells as the discrete boundaries and preserve their topology. For these reasons IPEP smoothed boundaries are very efficient for editing and segmenting small areas of the image. For the same reason we think that IPEP can be integrated in the segmentation process in order to drive segmentation steps. We are currently working on this problem.

\section{References}

[AAF95] E. Ahronovitz, J.P Aubert, and C. Fiorio. The star-topology: a topology for image analysis. In In 5th Discrete Geometry for Computer Imagery, Proceedings, pages 107-116, September 1995.

[BB96] Jean-Pierre Braquelaire and Luc Brun. Image segmentation with topological maps and inter-pixel representation. Technical report, LaBRI, Université Bordeaux I, 1996. submited.

[BD91] Jean-Pierre Braquelaire and Jean-Philippe Domenger. Intersection of discrete contours. In Proc. of Compugraphics'91, pages 14-23, 1991.

[BD96a] Jean-Pierre Braquelaire and Jean-Philippe Domenger. Representation of segmented images with discrete maps. Technical report, LaBRI, Université Bordeaux I, 1996. submited.

[BD96b] Luc Brun and Jean-Philippe Domenger. Segmentation. Technical report, LaBRI, Université Bordeaux I, 1996. submited.

[BF70] C.R. Brice and C.L. Fennema. Scene analysis using regions. Artificial intelligence, 1:205-226, 1970.

[Bie90] H. Bieri. Hyperimages - an alternative to the conventional digital images. In Eurographics'90 proceedings, pages 341-352, 1990.

[BV95] Jean-Pierre Braquelaire and Anne Vialard. Euclidean paths: a new representation of boundaries of discrete regions. Technical Report 1088-95, LaBRI, Université Bordeaux I, 1995. submited.

[CL90] Long-Wen Chang and Kuen-Long Leu. A fast algorithm for the restoration of images based on chain codes descriptions and its applications. Computer Vision, Graphics, and Image Processing, 50:296-307, 1990.

[Cor75] Robert Cori. Un code pour les graphes planaires et ses applications. Thèse d'état de l'université Paris VII, and Astŕisque 27, 1973 and 1975.

[Dom92] Jean-Philippe Domenger. Conception et implémentation du noyau graphique d'un environnement $2 D \frac{1}{2}$ d'édition d'images discrètes. $\mathrm{PhD}$ thesis, Univ. Bordeaux I, avril 1992.

[DR92] I. Debled and J.P. Reveilles. Un algorithme linéaire de polygonalisation des courbes discrètes. In Colloque Géométrie discrète en imagerie, pages 243-253, Grenoble, 1992. 
[Fio95] Christophe Fiorio. Approche interpixel en analyse d'images : une topologie et des algorithmes de segmentation. Thèse de doctorat, Université Montpellier II, 24 novembre 1995.

[Fra91] Jean Françon. Topologie de khalimsky et kovalevsky et algorithmes graphiques. In First Colloquium on Discrete Geometry in Computer Imagery, Strasbourg, September 1991.

[Fra95] Jean Françon. Topologie de khalimski-kovalevsky et algorithmique graphique. TSI, 14(10):119-121, 1995.

[Fre61] H. Freeman. On the encoding of arbitrary geometric configurations. IRE Trans., EC-10(2):260-268, 1961.

[Fre74] H. Freeman. Computer processing of line drawing images. ACM Comput. Surveys, 6(1):57-98, 1974 .

[GVT91] M. Gangnet and J.M. Van Thong. Robust boolean operations on 2d paths. In Proc, of COMPUGRAPHICS'91, pages 434-444,1991, 1991.

[KKM90a] E. Khalimsky, R. Kopperman, and P.R. Meyer. Boundaries in digital planes. Journal of applied Math, and Stocastic Analysis, 3:27-55, 1990.

[KKM90b] E. Khalimsky, R. Kopperman, and P.R. Meyer. Computer graphics and connected topologies on finite ordered sets. Topology and its Applications, $36: 1-17,1990$.

[Kov89] V.A. Kovalevsky. Finite topology as applied to image analysis. Computer Vision, Graphics, and Image Processing, 46:141-161, 1989.

[KR89] T.Y. Kong and A. Rosenfeld. Digital topology: Introduction and survey. Computer Vision, Graphics, and Image Processing, 48:357-393, 1989.

[Mer73] R.D. Merrill. Representation of contours and regions for efficient computer search. Communications of the $A C M, 16(2): 69-82,1973$.

[Mor69] P. Morse. Concepts of use in contour map processing. Communications of the ACM, 12(3):147-152, 1969.

[Pav82] T. Pavlidis. Algorithms for Graphics and Image Processing. Computer Sci., Washington, 1982.

[Rev91] J.P. Reveilles. Géométrie discrète, Calcul en nombres entiers et algorithmique. PhD thesis, Université Louis Pasteur, Strasbourg, 1991.

[RK82] A. Rosenfeld and A.C. Kak. Digital Picture Processing, volume 2. Academic Press, 1982.

[Via96] Anne Vialard. Geometrical parameters extraction from discrete paths. Technical report, LaBRI, Université Bordeaux I, 1996. submited. 


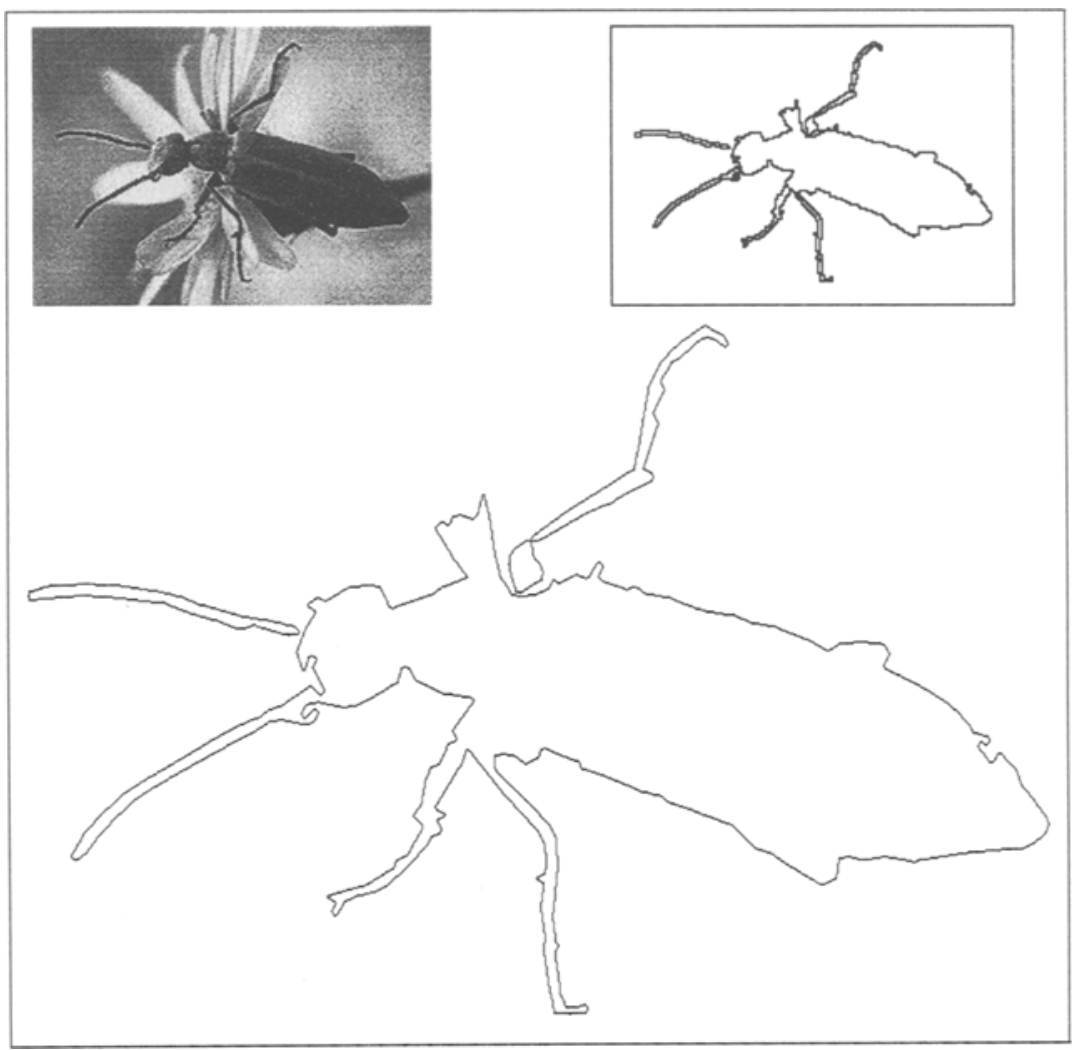

Fig. 6. The size of the original image is $139 \times 200$ pixels.

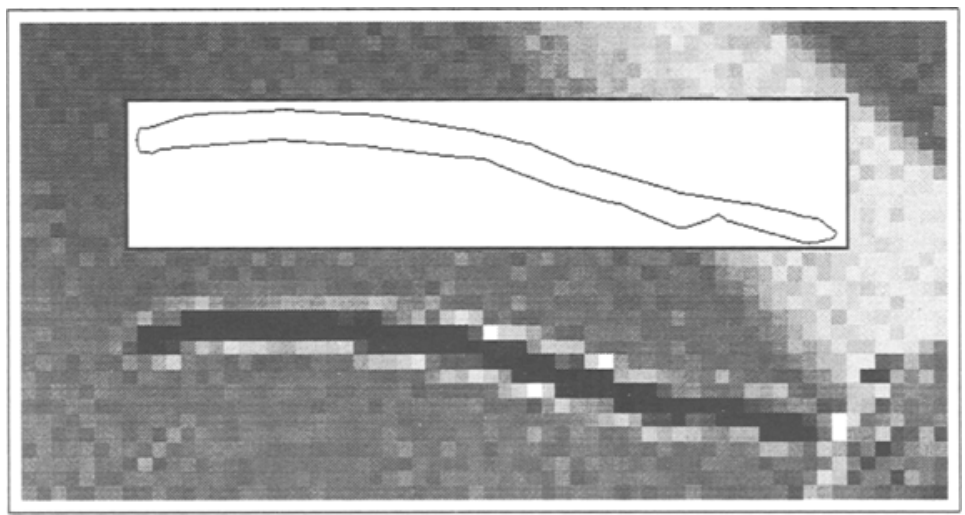

Fig. 7. In this image is displayed at scale 10 the upper horn of the insect with the drawing at the same scale of the IPEP smoothed boundary of the horn. 